Claremont Colleges

Scholarship@ Claremont

All HMC Faculty Publications and Research

HMC Faculty Scholarship

$11-1-1987$

\title{
The Bisection Method: Which Root?
}

Arthur T. Benjamin

Harvey Mudd College

\section{Recommended Citation}

Benjamin, Arthur T. "Bisection Method: Which Root?" The American Mathematical Monthly, Vol. 94, No. 9, pp. 861-863, November 1987.

This Article is brought to you for free and open access by the HMC Faculty Scholarship at Scholarship @ Claremont. It has been accepted for inclusion in All HMC Faculty Publications and Research by an authorized administrator of Scholarship @ Claremont. For more information, please contact scholarship@cuc.claremont.edu. 


\section{MAA}

The Bisection Method: Which Root?

Author(s): Arthur Benjamin

Source: The American Mathematical Monthly, Vol. 94, No. 9 (Nov., 1987), pp. 861-863

Published by: Mathematical Association of America

Stable URL: http://www.jstor.org/stable/2322818

Accessed: 10/06/2013 17:45

Your use of the JSTOR archive indicates your acceptance of the Terms \& Conditions of Use, available at

http://www.jstor.org/page/info/about/policies/terms.jsp

JSTOR is a not-for-profit service that helps scholars, researchers, and students discover, use, and build upon a wide range of content in a trusted digital archive. We use information technology and tools to increase productivity and facilitate new forms of scholarship. For more information about JSTOR, please contact support@ jstor.org. 


\title{
NOTES
}

\author{
Edited by Dennis Deturck, Richard Libera, and Anita E. Solow
}

\section{The Bisection Method: Which Root?}

\author{
ARTHUR BENJAMIN \\ Department of Mathematical Sciences, The Johns Hopkins University, Baltimore, MD 21218
}

Suppose that a continuous real-valued function $f$ has $2 N-1$ roots, $r_{1}, r_{2}, \ldots, r_{2 N-1}$, in an interval $(a, b)$ whose endpoints are not roots. If all the roots are simple, then $f(a) f(b)<0$ and the bisection method can be used to find a root of $f$. (Notice that when there are an even number of simple roots, $f(a) f(b)>0$, so that the bisection method does not apply over $(a, b)$.) It is the purpose of this note to show that the probability that the bisection method locates the $i$ th smallest root is zero when $i$ is even, assuming any continuous joint distribution for the roots. Furthermore, assuming the roots are independent and uniformly distributed on $(a, b)$, the probability that it finds the $i$ th smallest root is $1 / N$, when $i=$ $1,3, \ldots, 2 N-1$.

Recall that the bisection method locates a root in $(a, b)$ by first examining the sign of $f(c)$, where $c=(a+b) / 2$. If $f(c)=0$, a zero-probability event, then a root has been found. If $f(a) f(c)$ is negative, the process begins anew with $b$ replaced by $c$. Otherwise the process recommences with $a$ replaced by $c$. Ultimately, this procedure will find a root of $f$ to any desired accuracy by finding an interval of arbitrarily small length containing a root.

Let $(a, b)$ contain roots $r_{1}, r_{2}, \ldots, r_{2 N-1}$ where $a<r_{1}<r_{2}<\cdots<r_{2 N-1}<b$. Let $P(i)$ denote the probability that the bisection method finds root $r_{i}$, for $i=1,2, \ldots, 2 N-1$. Let $X$ denote the number of roots in $(a, c)$.

We first prove, by induction on $N$, that $P(i)=0$ when $i$ is even. When $N=1$, the assertion is immediate. Assume the assertion is true for any interval $(\alpha, \beta)$ which contains $2 j-1$ roots, $j=1,2, \ldots, N$. Suppose $(a, b)$ has $2 N+1$ roots. There are four cases to consider:
(i) $X=2 N+1$
(ii) $X=0$
(iii) $X=2 j-1$ for some $j \in\{1, \ldots, N\}$
(iv) $X=2 j$

for some $j \in\{1, \ldots, N\}$.

Notice that the bisection method will always search next the half of the interval that contains an odd number of roots. In case (i) or (ii), the bisection method will search the interval that contains all the roots and will eventually reach case (iii) or (iv). In case (iii), the bisection method will replace $b$ with $c$ and begin again. Here, $P(i)=0$ for $i>2 j-1$, and by our induction hypothesis $P(i)=0$ when $i=$ $2,4, \ldots, 2 j-2$. Thus $P(i)=0$ for $i=2,4, \ldots, 2 N$. In case (iv), $a$ will be replaced by $c$ and the process begins anew. Here $P(i)=0$ for $i \leqslant 2 j$ and by our induction hypothesis, $P(i)=0$ for $i=2 j+k$ for all even $k$. Thus $P(i)=0$ for $i=$ $2,4, \ldots, 2 N$, and the induction is complete. 
Now suppose that the $2 N-1$ roots of $(a, b)$ are independently distributed according to a uniform distribution. We will prove by induction on $N$ that $P(i)=1 / N$ for $i=1,3, \ldots, 2 N-1$. When $N=1$, the assertion is obviously true. Assume for $j=1, \ldots, N$ that if any interval $(\alpha, \beta)$ contains $2 j-1$ roots uniformly distributed, the $P(i)=1 / j$ for $i=1,3, \ldots, 2 j-1$. Now suppose $(a, b)$ contains $2 N+1$ roots. From the law of total probability, we have

$$
P(i)=\sum_{k=0}^{2 N+1} P(i \mid X=k) P(X=k) .
$$

Since the roots are independent and uniformly distributed, then from binomial probability, the probability that exactly $k$ roots be in $(a, c)$ is

$$
P(X=k)=\left(\begin{array}{c}
2 N+1 \\
k
\end{array}\right)\left(\frac{1}{2}\right)^{k}\left(\frac{1}{2}\right)^{2 N+1-k}=\left(\begin{array}{c}
2 N+1 \\
k
\end{array}\right)\left(\frac{1}{2}\right)^{2 N+1} .
$$

Thus

$$
P(i)=\sum_{k=0}^{2 N+1} P(i \mid X=k)\left(\begin{array}{c}
2 N+1 \\
k
\end{array}\right)\left(\frac{1}{2}\right)^{2 N+1} .
$$

Clearly, $P(i \mid X=0)=P(i \mid X=2 N+1)=P(i)$. Therefore,

$$
P(i)=\sum_{k=1}^{2 N}\left(P(i \mid X=k)\left(\begin{array}{c}
2 N+1 \\
k
\end{array}\right)\left(\frac{1}{2}\right)^{2 N+1}\right)+2\left(\frac{1}{2}\right)^{2 N+1} P(i)
$$

or equivalently,

$$
\begin{aligned}
\left(1-4^{-N}\right) P(i)= & \sum_{\substack{k=1 \\
k \text { odd }}}^{2 N-1} P(i \mid X=k)\left(\begin{array}{c}
2 N+1 \\
k
\end{array}\right)\left(\frac{1}{2}\right)^{2 N+1} \\
& +\sum_{\substack{k=2 \\
k \text { even }}}^{2 N} P(i \mid X=k)\left(\begin{array}{c}
2 N+1 \\
k
\end{array}\right)\left(\frac{1}{2}\right)^{2 N+1} .
\end{aligned}
$$

Now, if the value $k$ of $X$ is odd, then the interval $(a, c)$ has $(k+1) / 2$ odd roots. If $k$ is even, then the interval $(c, b)$ has $(2 N+2-k) / 2$ odd roots. Furthermore, given $k$, the distribution of the roots remains uniform over whichever interval contains an odd number. Therefore, by our induction hypothesis,

$$
P(i \mid X=k)=\left\{\begin{array}{cl}
\frac{2}{k+1}, & \text { if } k \text { is odd and } i \leqslant k \\
\frac{2}{2 N+2-k}, & \text { if } k \text { is even and } k+1 \leqslant i \\
0, & \text { otherwise. }
\end{array}\right.
$$


Thus, (1) becomes

$$
\begin{aligned}
& \left(1-4^{-N}\right) P(i)= \\
& \left(\frac{1}{2}\right)^{2 N+1}\left[\sum_{\substack{k=i \\
k \text { odd }}}^{2 N-1}\left(\begin{array}{c}
2 N+1 \\
k
\end{array}\right)\left(\frac{2}{k+1}\right)+\sum_{\substack{k=2 \\
k \text { even }}}^{i-1}\left(\begin{array}{c}
2 N+1 \\
k
\end{array}\right)\left(\frac{2}{2 N+2-k}\right)\right] .
\end{aligned}
$$

Substituting $j$ for $k-1$ gives us:

$$
\begin{aligned}
& \left(1-4^{-N}\right) P(i)= \\
& \begin{aligned}
\left(\frac{1}{2}\right)^{2 N} & {\left[\sum_{\substack{k=i \\
k \text { odd }}}^{2 N-1} \frac{(2 N+1) !}{(k+1) !(2 N+1-k) !}+\sum_{\substack{j=1 \\
j \text { odd }}}^{i-2} \frac{(2 N+1) !}{(j+1) !(2 N+1-j) !}\right] } \\
& =4-N \sum_{\substack{j=1 \\
j \text { odd }}}^{2 N-1} \frac{(2 N+1) !}{(j+1) !(2 N+1-j) !},
\end{aligned}
\end{aligned}
$$

which no longer depends on $i$. Therefore, $P(i)=1 /(N+1)$. The induction is complete.

More generally, through the probability integral transformation, we obtain the same results when the roots are independent and identically distributed over $(a, b)$ according to an arbitrary positive continuous distribution $f$, and when the point $c$ for which the sign of $f(c)$ is tested is the median of $f$ restricted to the "current" subinterval $(\alpha, \beta)$.

\title{
Spaces with Locally Compact Completions are Compact
}

\author{
ALFONSO VilLANI \\ Department of Mathematics, University of Catania, Catania, Italy
}

In this note we characterize compactness in metrizable spaces in a way that has apparently not been pointed out elsewhere. We refer the reader to [4] for terminology. In particular, given a metrizable space $X$, we say that a metric $d$ on $X$ is compatible provided that $d$ generates the topology of $X$; also, we say that a metric space $(\hat{X}, \hat{d})$ is a metric completion of $X$ provided that $(\hat{X}, \hat{d})$ is the metric completion of some metric space $(X, d)$, with $d$ a compatible metric on $X$.

THEOREM 1. A metrizable space $X$ is compact if and only if every metric completion of $X$ is locally compact.

The key, in the proof of Theorem 1, is the following well-known result on extending metrics, due to Hausdorff [2], and rediscovered by Bing [1]; see also [4], Problem 22.E. 